\title{
Влияние конструкции активной области и волновода на характеристики лазеров на основе структур квантовые ямы-точки InGaAs/GaAs
}

\author{
(С) Ю.М. Шерняков ${ }^{1,2}$, Н.Ю. Гордеев ${ }^{1}$, А.С. Паюсов ${ }^{1}$, А.А. Серин ${ }^{1}$, Г.О. Корнышов ${ }^{2}$, А.М. Надточий ${ }^{3}$, \\ М.М. Кулагина ${ }^{1}$, С.А. Минтаиров ${ }^{1,2}$, Н.А. Калюжный ${ }^{1,2}$, М.В. Максимов ${ }^{2}$, А.Е. Жуков ${ }^{3}$ \\ ${ }^{1}$ Физико-технический институт им. А.Ф. Иоффре Российской академии наук, \\ 194021 Санкт-Петербург, Россия \\ ${ }^{2}$ Санкт-Петербургский Академический университет им. Ж.И. Алфёрова, \\ 194021 Санкт-Петербург, Россия \\ ${ }^{3}$ Национальный исследовательский университет „Высшая школа экономики“, \\ 190008 Санкт-Петербург, Россия \\ E-mail: yuri.shernyakov@mail.ioffe.ru
}

Поступила в Редакцию 30 октября 2020 г.

В окончательной редакции 1 ноября 2020 г.

Принята к публикации 1 ноября 2020 г.

Исследованы торцевые лазеры с активной областью на основе нового типа наногетероструктур InGaAs/GaAs переходной размерности, именно структур квантовые яма-точки, занимающих по своим свойствам промежуточное положение между квантовыми ямами и квантовыми точками. Показано, что скорость коротковолнового сдвига линии лазерной генерации при уменьшении длины резонатора замедляется как с увеличением числа слоев в активной области, так и с возрастанием фактора оптического ограничения. В лазере с 10 слоями квантовых яма-точек линия лазерной генерации остается в пределах основного оптического перехода вплоть до самых малых длин резонатора (100 мкм). В приборах с одним слоем квантовых яма-точек и (или) малым фактором оптического ограничения при уменьшении длины резонатора до $\leq 200$ мкм происходит переключение лазерной генерации с основного состояния квантовых яма-точек непосредственно на состояния волновода, минуя возбужденные состояния. Данный эффект не наблюдался в лазерах на квантовых ямах и квантовых точках и может быть следствием аномально малой плотности возбужденных состояний в квантовых яма-точках.

Ключевые слова: квантовые яма-точки, полупроводниковые лазеры, конструкция волновода, полосковые лазеры.

DOI: 10.21883/FTP.2021.03.50604.9547

\section{1. Введение}

В современных полупроводниковых лазерах широко используется активная область на основе квантовых ям (КЯ) или квантовых точек (КТ). Оба типа квантоворазмерных гетероструктур имеют свои достоинства и недостатки, определяемые их структурными и оптическими свойствами. Квантовые ямы имеют узкий спектр усиления и высокое максимальное усиление. К недостаткам КЯ можно отнести большое значение фактора уширения спектральной линии ( $\alpha$-фактора), ограничение по доступным спектральным диапазонам, а также необходимость использования достаточно сложной технологии компенсации упругих напряжений при эпитаксиальном синтезе относительно большого количества КЯ (более 3 для оптического диапазона $980 \mathrm{Hм}$ ). КТ имеют широкий спектр усиления и малое значение $\alpha$-фактора, а также позволяют достичь рекордно низких значений пороговой плотности тока и его высокой температурной стабильности. За счет эффективной релаксации упругих напряжений возможен последовательный синтез до 20 слоев КТ высокого оптического и структурного совершенства. Недостатком КТ является невысокое максимальное усиление на один слой. Для лазеров на основе квантовых точек характерны такие явления, как коротковолновый перескок длины волны генерации, одновременная генерация на нескольких оптических переходах, выраженное нелинейное насыщение усиления. Как следствие, для применений, в которых требуются высокие значения оптического усиления, на сегодня более предпочтительным является использование квантовых ям. В то же время свободный транспорт носителей заряда вдоль слоя квантовой ямы приводит к тому, что носители могут беспрепятственно диффундировать к центрам безызлучательной рекомбинации на открытых поверхностях, увеличивая пороговый ток или делая лазерную генерацию (например, в микролазерах) вообще невозможной. Квантовые точки и квантовые ямы различаются динамикой захвата носителей, а также динамикой спектров усиления и поглощения, что существенно сказывается на работе лазеров в режиме прямой модуляции и в режиме синхронизации мод. В частности, лазеры на квантовых точках позволяют получить меньшие длительности импульсов, но и меньшие мощности по сравнению с квантовыми ямами. 
Таким образом, весьма перспективной и актуальной является задача создания с помощью эпитаксиальных технологий и принципов зонной инженерии нового типа наногетероструктур, которые одновременно обладали бы рядом преимуществ, присущих как квантовым ямам, так и квантовым точкам. В частности, желательно совместить высокие значения оптического усиления, характерные для квантовых ям, со слабой чувствительностью к дефектам и к поверхностной рекомбинации, присущей квантовым точкам. Недавно мы представили новый тип наноструктур с переходной размерностью $0 \mathrm{D} / 2 \mathrm{D}$, которые образуются при осаждении нескольких монослоев (ML) $\operatorname{In}_{x} \mathrm{Ga}_{1-x} \mathrm{As}(0.3<x<0.5)$ на вицинальные подложки GaAs методом газофазной эпитаксии из металлоорганических соединений (МОГФЭ) [1]. Такие наноструктуры представляют собой сверхплотный массив островков $\operatorname{In}_{x} \mathrm{Ga}_{1-x}$ As в остаточной КЯ $\operatorname{In}_{y} \mathrm{Ga}_{1-y} \mathrm{As}$ $(x>y)$ и в дальнейшем упоминаются как квантовые яма-точки (KЯT, QWD). Поскольку содержание индия в $\mathrm{KT} \operatorname{In}_{x} \mathrm{Ga}_{1-x}$ As и высота заметно больше по сравнению с окружающим их слоем $\operatorname{In}_{y} \mathrm{Ga}_{1-y} \mathrm{As}$, в них происходит локализация носителей, однако энергия локализации оказывается меньше, чем в КТ, полученных в режиме роста Странского-Крастанова. Использование КЯТ в качестве активной области микродисковых лазеров позволило реализовать рекордно высокую для данного типа приборов оптическую мощность излучения 18 мВт [2], а также осуществить передачу информации со скоростью 10 Гб/с [3]. В то же время оптическое усиление, которое непосредственно влияет на ряд ключевых характеристик светоизлучающих приборов, недостаточно изучено.

В данной работе мы исследуем зависимость основных лазерных характеристик от числа слоев КЯТ в активной области и от конструкции волновода.

\section{2. Экспериментальные образцы и методы исследования}

Для описания оптического усиления нами используется зависимость его максимального значения, экспериментально определяемого как сумма внутренних оптических потерь и потерь на выход излучения, от пороговой плотности тока. При этом с увеличением потерь на выход излучения и соответственно пороговой плотности тока происходит коротковолновый сдвиг длины волны линии лазерной генерации. При значительном росте потерь на выход излучения усиление в основном состоянии может оказаться недостаточным для преодоления суммарных потерь, что приводит к скачкообразному переключению лазерной генерации на возбужденные состояния. Данные процессы наиболее ярко проявляются в лазерах на квантовых точках [4], но при высоких потерях наблюдаются и в лазерах на квантовых ямах [5]. Сдвиг длины волны генерации с увеличением суммарных потерь определяется модальным усилением, т.е. произведением материального усиления активной области и фактора оптического ограничения, зависящего от конструкции волновода. Соответственно на характеристики усиления лазера оказывают влияние как тип активной области, так и особенности ее конструкции (количество рядов наноструктур), а также конструкция лазерного волновода, определяющая величину фактора оптического ограничения активной области.

Лазерные гетероструктуры синтезировали методом газофазной эпитаксии из металлоорганических соединений (МОГФЭ) на разориентированных подложках $n$-GaAs. Исследовались лазерные диоды на основе трех типов гетероструктур: с волноводом шириной $W_{w g}=0.45$ мкм (narrow waveguide, NWG), с волноводом шириной $W_{w g}=0.78$ мкм (broad waveguide, BWG) и волноводом на основе связанных резонаторов (coupled large optical cavity, CLOC) [6], ширина активной части которого составляла 1.34 мкм. Последовательность слоев для каждой из лазерных гетероструктур представлена на рис. 1. Активная область, состоящая из $N$ слоев КЯТ $(N=1-10)$, разделенных барьером GaAs 40 нм, помещалась в центр волновода. Каждый из слоев КЯТ формировался осаждением 8 монослоев $\operatorname{In}_{0.4} \mathrm{Ga}_{0.6} \mathrm{As}$.

По стандартной постростовой технологии были изготовлены лазеры с мелкой мезой и широким полоском ( $W=50$ или 100 мкм), с длиной резонатора $L$, варьируемой от 100 мкм до 4 мм. На лазерные грани покрытия не наносились. Образцы напаивались на медные теплоотводы $p$-слоями вниз при помощи индиевого припоя. Теплоотводы монтировались на термостабилизированный элемент Пельтье. Для измерения ваттамперных характеристик использовался калиброванный

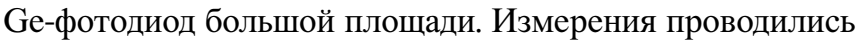
в импульсном режиме (длительность импульсов 0.3 мкс, частота следования 2 кГц) при комнатной температуре. Из ватт-амперных характеристик определялись зависимости плотности порогового тока $\left(J_{\text {th }}\right)$ и обратной дифференциальной эффективности $\left(1 / \eta_{d}\right)$ от длины резонатора. Спектры фотоотклика при волноводном поглощении были получены при освещении торца лазерного чипа, при этом использовалось неполяризованное излучение лампы накаливания, прошедшее через монохроматор. Регистрация фототока проводилась в стандартной схеме синхронного детектирования без внешнего смещения.

\section{3. Результаты и обсуждение}

На рис. 2 представлены спектры электролюминесценции лазеров с уширенным волноводом (BWG) и активной областью на основе 1,2 и 10 слоев КЯТ. Длина резонатора была выбрана равной 100 мкм для подавления лазерной генерации вплоть до высоких токов инжекции, а также чтобы самопоглощение в активной области как можно меньше влияло на форму спектров электролюминесценции. Видно, что ширина спектров излучения возрастает с ростом тока накачки в основном 

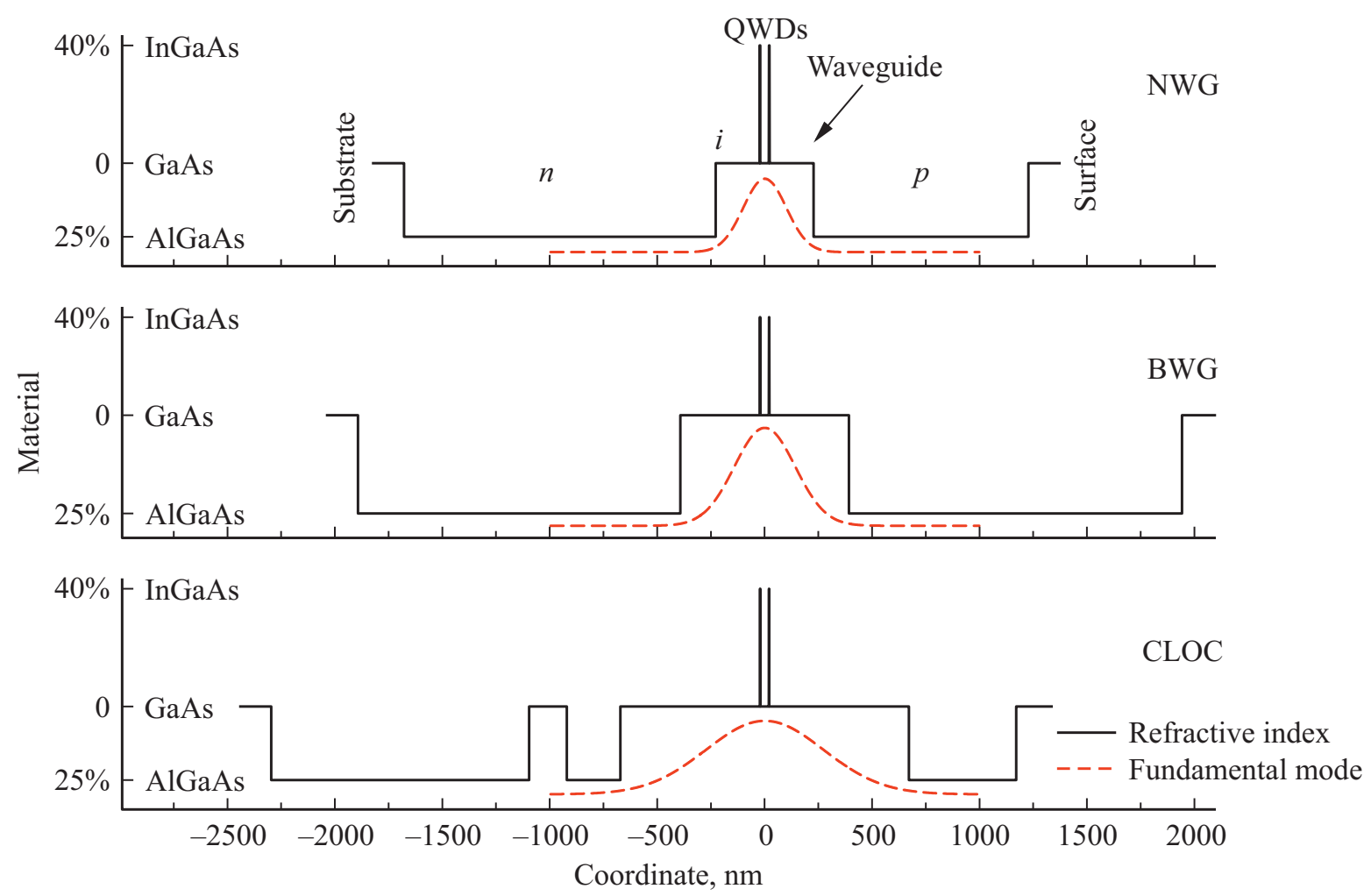

Рис. 1. Схематическое изображение волноводов лазерных структур. Штриховой линией показан профиль фундаментальной моды.

за счет увеличения интенсивности в коротковолновой части спектра. Уширение спектров происходит тем сильнее, чем меньше число слоев КЯТ, поскольку ток инжекции, приходящийся на один слой КЯТ, возрастает обратно пропорционально числу слоев.

На рис. 2 также представлены спектры фотоотклика при волноводном поглощении, на которых хорошо различимы неоднородно уширенные линии основного (длина волны $\lambda \approx 1.02-1.1$ мкм) и возбужденного $(\lambda \approx 0.94-0.97$ мкм $)$ состояний КЯТ, а также состояния GaAs-волновода. В отличие от спектра электролюминесценции, спектр фотоотклика позволяет достаточно точно определить положения основного и возбужденных состояний, однако оценить плотность состояний КЯТ по его интенсивности можно только приблизительно.

Характерной особенностью спектров электролюминесценции является то, что в них слабо проявляются полосы излучения, связанные с возбужденными состояниями. Так, например, в случае структуры с 1 слоем КЯТ при больших токах инжекции состояния волновода GaAs заполняются носителями и интенсивность его электролюминесценции оказывается выше, чем интенсивность электролюминесценции основного состояния КЯТ, однако даже при такой накачке интенсивность возбужденного состояния остается относительно слабой.

На рис. 2 точками показаны зависимости длины волны генерации от пороговой плотности тока в лазерах различной длины. Пороговая плотность тока однозначно связана с длиной лазера - чем короче резонатор, тем больше потери на выход излучения и тем выше пороговая плотность тока. Длины волн генерации соответствуют спектральному положению максимумов спектров усиления при росте тока инжекции, которые, как правило, не совпадают с максимумами спектров электролюминесценции при том же токе. В лазерных диодах с одним слоем КЯТ линия генерации возникает на длинноволновом крае спектра электролюминесценции, измеренного при слабой накачке, и с уменьшением длины лазера достаточно быстро смещается на его коротковолновый край, соответствующий спектральной области между основным и возбужденными состояниями. При дальнейшем уменьшении длины резонатора до значений $<200$ мкм происходит переключение лазерной генерации с состояний КЯТ на состояния в волноводе GaAs вблизи 0.89 мкм (рис. 2,a), поскольку усиления одного слоя КЯТ уже недостаточно, чтобы преодолеть потери на выход излучения. Тот факт, что лазерная генерация переключается непосредственно на состояния волновода, минуя возбужденные состояния, позволяет предположить, что плотность возбужденных состояний в КЯТ меньше плотности основных, в отличие от случаев КЯ и КТ.

При увеличении числа слоев КЯТ до 2 коротковолновый сдвиг линии лазерной генерации с ростом накачки происходит медленнее, а перескок на состояния GaAs не наблюдается. Мы полагаем, это связано с тем, что необходимое для преодоления суммарных потерь модальное усиление достигается при меньших токах за 

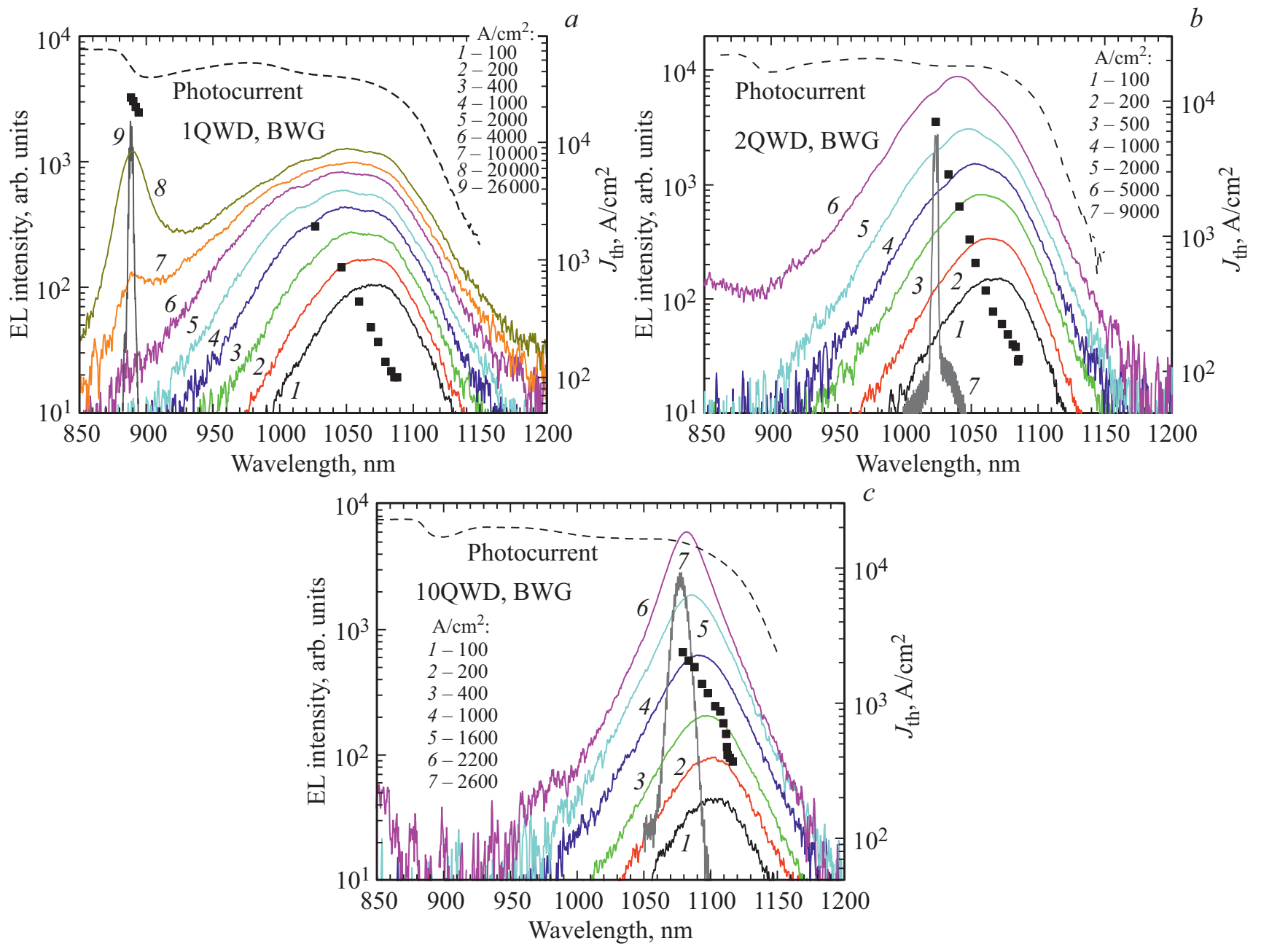

Рис. 2. Спектры электролюминесценции (EL) и лазерной генерации диодов длиной 0.1 мм с волноводом шириной 0.78 мкм (BWG) и активной областью на основе $1(a), 2(b)$ и $10(c)$ слоев КЯТ. Точками показаны зависимости длины волны генерации от пороговой плотности тока в лазерах различной длины. Штриховой линией показаны спектры фотоотклика при волноводном поглощении.

счет в 2 раза большего фактора оптического ограничения. При токе инжекции $>5 \mathrm{\kappa A} \cdot \mathrm{cm}^{-2}$ линия генерации сдвигается в коротковолновую сторону вплоть до $1.02-1.03$ мкм, так же, как и в случае лазера с одним слоем КЯТ, не доходя до спектральной области, соответствующей переходам на возбужденные состояния.

В случае лазерных диодов с активной областью на основе 10 слоев КЯТ коротковолновый сдвиг линии генерации практически не наблюдается вплоть до пороговых плотностей тока $\sim 1 \mathrm{\kappa A} \cdot \mathrm{cm}^{-2}$. При дальнейшем увеличении пороговой плотности тока (т.е. уменьшении длины резонатора) начинается относительно медленный коротковолновый сдвиг длины волны лазерной генерации. При токе инжекции $2.6 \mathrm{\kappa A} \cdot \mathrm{cm}^{-2}$ длина волны генерации сдвигается в коротковолновую область до 1.08 мкм, по-прежнему оставаясь в пределах линии основного состояния.

Кроме числа слоев КЯТ в активной области на поведение длины волны лазерной генерации при изменении длины резонатора большое влияние оказывает конструкция волновода, которая определяет фактор оптического ограничения каждого из слоев и соответственно модальное усиление. В случае исследуемых лазерных диодов влияние волновода иллюстрирует рис. 3 , на котором представлены зависимости длины волны генерации, пороговой плотности тока и обратной дифференциальной квантовой эффективности от длины резонатора для приборов с одинаковой активной областью на основе 2 слоев КЯТ и различными конструкциями волновода: NWG, BWG и CLOC. NWG имеет наибольший фактор оптического ограничения, а CLOC - наименьший.

В лазерах всех типов с длиной резонатора $L=4 \mathrm{Mм}$ длины волн генерации (рис. 3, $a$ ) очень близки и находятся в диапазоне $1085-1087$ нм. При уменьшении $L$ длины волн генерации смещаются в коротковолновую сторону, причем скорость смещения максимальна для лазера CLOC и минимальна для лазера NWG. Особенно заметен данный эффект при $L<0.5$ мм. Когда $L$ стано- 

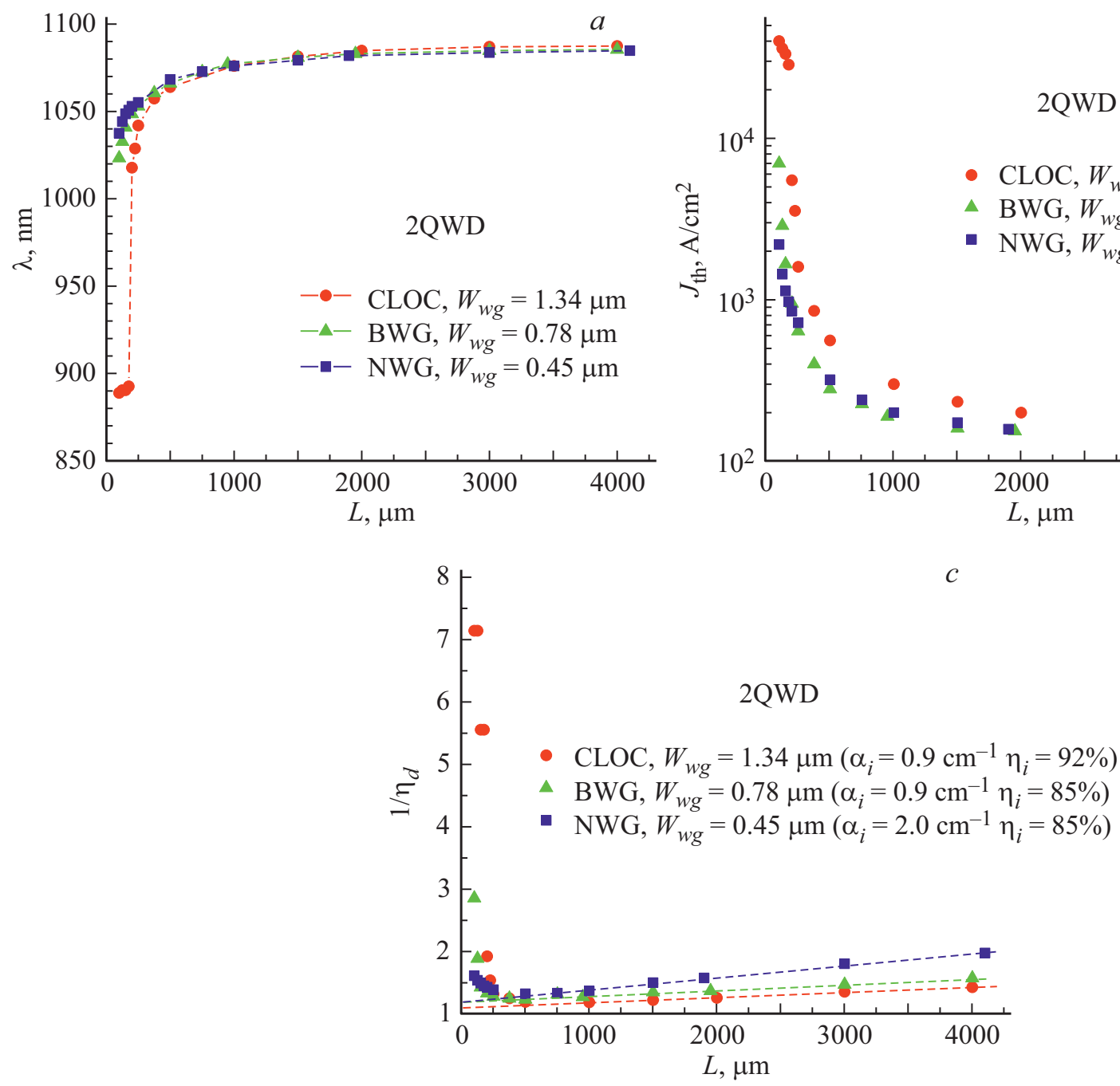

Рис. 3. Зависимости длины волны лазерной генерации $(a)$, пороговой плотности тока $(b)$ и обратной дифференциальной квантовой эффективности $(c)$ от длины резонатора для приборов с одинаковой активной областью на основе двух слоев КЯТ и различными конструкциями волновода: CLOC, BWG и NWG.

вится < 200 мкм, в лазере CLOC возникает генерация через объемный материал волновода GaAs. Отметим, что перескок лазерной генерации на состояния $\mathrm{GaAs}$ волновода происходит во всех исследованных типах лазеров с 1 слоем КЯТ, в то время как в лазере с 2 слоями КЯТ - только в лазере CLOC.

Пороговые плотности тока, а также их зависимости от длины резонатора (рис. $3, b)$ очень слабо различаются в случае лазеров с расширенным (BWG) и узким (NWG) волноводами $\left(\sim 120 \mathrm{~A} \cdot \mathrm{cm}^{-2}\right.$ для $\left.L=4 \mathrm{MM}\right)$. В лазере на основе связанных волноводов (CLOC) пороговая плотность несколько больше $\left(\sim 150 \mathrm{~A} \cdot \mathrm{cm}^{-2}\right.$ для $\left.L=4 \mathrm{MM}\right)$.

Лазер CLOC имеет наибольшую по сравнению с лазерами BWG и NWG дифференциальную квантовую эффективность $\left(\eta_{d}\right)$ в диапазоне длин резонатора $0.5-4$ мм. В длинных диодах при $L=4$ мм дифференциальная квантовая эффективность составляет 70, 64 и 50\% в лазерах CLOC, BWG и NWG соответственно. Во всех

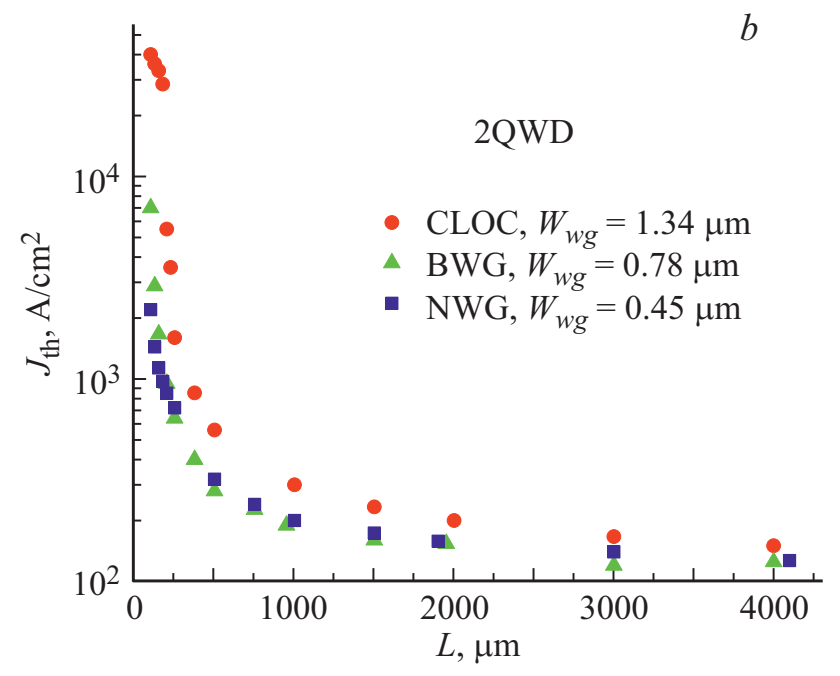

CLOC, $w_{w g}=1.34 \mu \mathrm{m}$

BWG, $W_{w g}=0.78 \mu \mathrm{m}$

- NWG, $W_{w g}=0.45 \mu \mathrm{m}$

(1)$$
\text { . }
$$

(1)

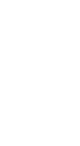


Значения плотности тока прозрачности $\left(J_{\mathrm{tr}}\right)$ и параметра модового усиления $\left(G_{0}\right)$, вычисленные на двух логарифмических участках зависимости модального усиления от плотности тока.

\begin{tabular}{c|c|c|c|c|c|r|r|r}
\hline & \multicolumn{3}{|c|}{ BWG, $W_{w g}=0.78 \mu \mathrm{m}$} & \multicolumn{3}{c}{2 QWD } \\
\hline Активная область & 1 QWD & 2 QWD & 5 QWD & 10 QWD & Конструкция волновода & CLOC & BWG & NWG \\
\hline$J_{\mathrm{tr} 1}, \mathrm{~A} \cdot \mathrm{cm}^{-2}$ & 70 & 100 & 190 & 300 & $J_{\mathrm{tr} 1}, \mathrm{~A} \cdot \mathrm{cm}^{-2}$ & 120 & 100 & 100 \\
$J_{\mathrm{tr} 2}, \mathrm{~A} \cdot \mathrm{cm}^{-2}$ & 220 & 400 & 550 & 800 & $J_{\mathrm{tr} 2}, \mathrm{~A} \cdot \mathrm{cm}^{-2}$ & 400 & 400 & 400 \\
$G_{01}, \mathrm{~cm}^{-1}$ & 14 & 20 & 26 & 22 & $G_{01}, \mathrm{~cm}^{-1}$ & 15 & 20 & 21 \\
$G_{02}, \mathrm{~cm}^{-1}$ & 35 & 75 & 95 & 115 & $G_{02}, \mathrm{~cm}^{-1}$ & 40 & 75 & 95
\end{tabular}

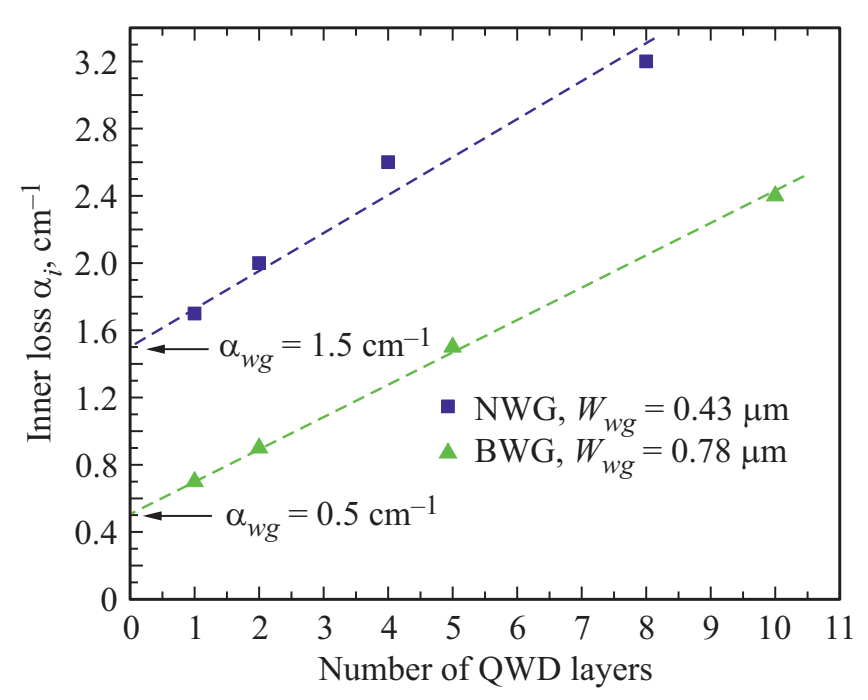

Рис. 4. Зависимость внутренних потерь от количества слоев КЯТ в активной области для лазеров с расширенным (BWG) и узким (NWG) волноводом.

граней, принимаемый равным 0.3. Из этой зависимости для всех исследуемых лазеров были определены внутренние потери.

В лазере BWG внутренние потери растут от 0.7 до $2.4 \mathrm{~cm}^{-1}$ при увеличении числа слоев КЯТ от 1 до 10 , а в лазере NWG - от 1.5 до $3.2 \mathrm{~cm}^{-1}$ при увеличении числа слоев КЯТ от 1 до 8 (рис. 4). Зависимость внутренних потерь от числа слоев позволила определить величину потерь, приходящуюся на 1 слой КЯТ $\left(0.2 \mathrm{~cm}^{-1}\right.$ в лазерах как BWG, так и NWG), а также на волновод и прилегающие слои эмиттеров. Последние в лазере BWG оказались равными $0.5 \mathrm{~cm}^{-1}$, а в NWG заметно выше и составляют $1.5 \mathrm{~cm}^{-1}$, что можно объяснить большим проникновением лазерной моды в легированные эмиттеры.

Линейная зависимость $1 / \eta_{d}$ от $L$ при больших длинах резонатора свидетельствует о независимости внутренних потерь и внутреннего квантового выхода от длины резонатора. Однако при меньших длинах резонатора (при $L<0.5$ мм) дифференциальная квантовая эффективность падает, и зависимость $1 / \eta_{d}$ от $L$ существенно отклоняется от линейной (рис. 3). В коротких лазерных диодах с большими потерями на зеркалах для достиже- ния генерации требуется большее оптическое усиление. Поэтому уменьшение длины резонатора приводит к возрастанию плотности порогового тока и заселению вышележащих энергетических состояний. Вследствие этого неизбежно возрастают и внутренние потери, связанные с поглощением на свободных носителях. Для вычисления внутренних потерь во всем диапазоне длин резонатора $(L=0.1-4 \mathrm{Mм})$ мы по-прежнему использовали вышеприведенную формулу [7]. При этом мы предполагали внутреннюю дифференциальную квантовую эффективность независимой от длины резонатора и равной значению, определенному при больших длинах резонатора, а внутренние потери полагали зависящими от длины резонатора. На основании полученных таким образом значений внутренних потерь $\alpha_{i}$ и экспериментальной зависимости пороговой плотности тока от длины резонатора определено модальное оптическое усиление $G=\alpha_{m}+\alpha_{i}$ и его зависимость от плотности тока накачки (рис. 5). Необходимо отметить, что используемый нами метод определения зависимости модального усиления от тока имеет определенные ограничения. Максимальное измеряемое усиление ограничено минимальной длиной резонатора, при которой достигается лазерная генерация, а количество экспериментальных значений усиления при больших токах определяется числом исследованных лазеров разной длины, близкой к минимальной.

Обычно зависимость усиления от плотности тока в структурах с квантовыми ямами описывают логарифмической функцией $G=G_{0} \ln \left(J / J_{\text {tr }}\right)$, где $G_{0}$ - параметр модового усиления, $J_{\mathrm{tr}}$ - плотность тока прозрачности. В случае активной области, состоящей из КЯТ, как видно из рис. 5, зависимость усиления от плотности тока во всем интервале плотностей тока отличается от логарифмической. Тем не менее для каждого лазера можно выделить два участка с логарифмической зависимостью $G$ от $J$. Параметры $J_{\operatorname{tr}}$ и $G_{0}$ для этих участков (с индексами 1 и 2) представлены в таблице.

В лазерных диодах с волноводом BWG с 1, 2, 5 и 10 слоями КЯТ плотность тока прозрачности на начальном участке зависимости $G(J)$ составила 70,100 , 190 и $300 \mathrm{~A} \cdot \mathrm{cm}^{-2}$ соответственно. Параметр модового усиления $G_{01}$ возрастает от 14 до $26 \mathrm{~cm}^{-1}$ с ростом числа слоев от 1 до 5, а затем в лазере с 10 слоями КЯТ несколько снижается до значения $22 \mathrm{~cm}^{-1}$. Умень- 


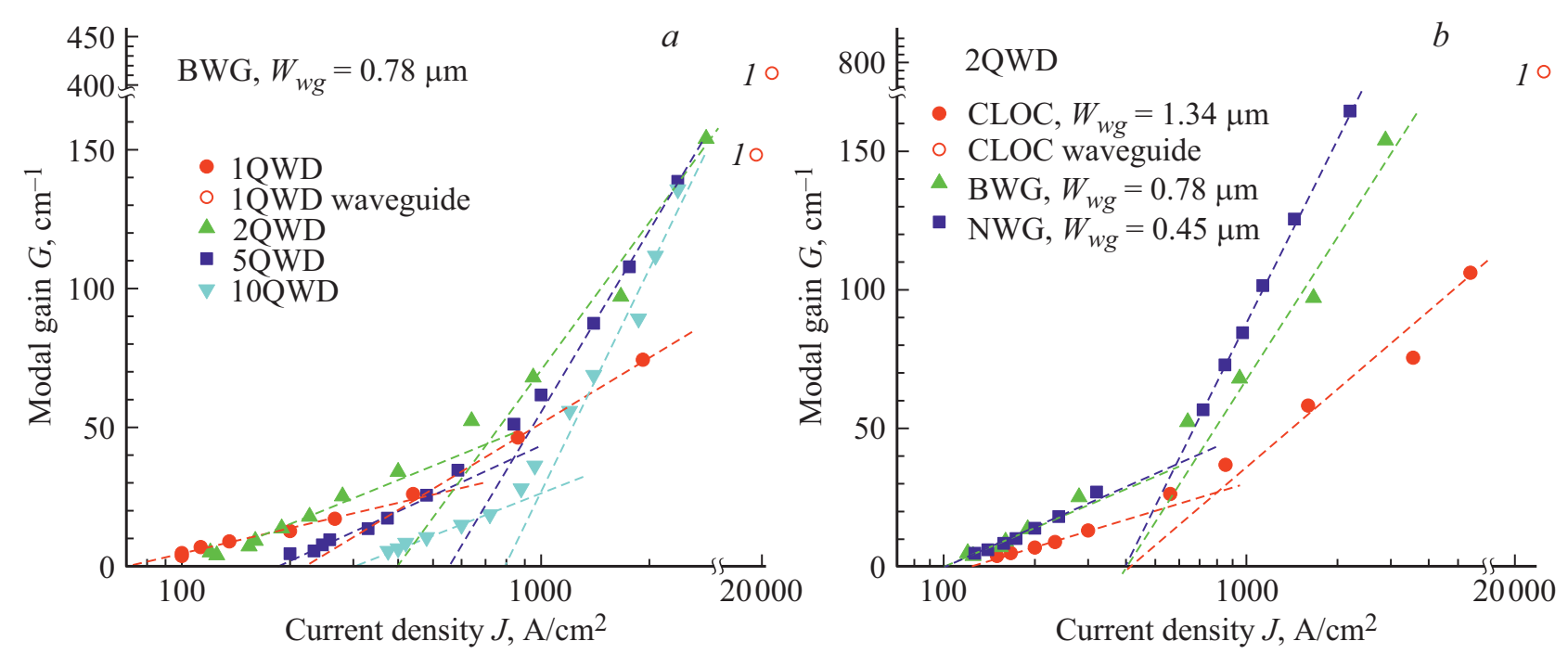

Рис. 5. Зависимости модального усиления от плотности тока для лазеров с толщиной волновода 0.78 мкм (BWG) и различным числом слоев КЯТ в активной области $(a)$ и для лазеров с активной областью на основе двух слоев КЯТ и различной толщиной волновода $(b)$. На обоих рисунках точки, соответствующие генерации через состояния волновода, отмечены пустыми кружками.

шение $G_{01}$ для лазера с 10 слоями КЯТ при малых токах может объясняться неоднородной прокачкой многослойной активной области. Для структур на основе КЯ экспериментально было показано $[8,9]$, что плотность носителей заряда в отдельных слоях активной области уменьшается с их отдалением от р-области прибора. Аналогичные результаты были получены для структур на основе КТ посредством моделирования [10]. Это происходит из-за меньшей подвижности дырок по сравнению с электронами и их более вероятным захватом в слои КТ, находящиеся рядом с $p$-областью, по сравнению с более удаленными от р-области слоями (захват электронов в слои КТ намного меньше зависит от расстояния относительно $n$-области).

На втором логарифмическом участке, наблюдаемом при больших плотностях тока, параметр модового усиления $G_{02}$ в 10 -слойном лазере не уменьшается по сравнению с 5-слойным и таким образом $G_{02}$ монотонно возрастает от 35 до $115 \mathrm{~cm}^{-1}$ с ростом числа слоев от 1 до 10. Данный факт может свидетельствовать о достижении режима однородной прокачки всей активной области лазера с 10 слоями КЯТ.

На рис. 5, $b$ показано влияние конструкции волновода (NWG, BWG и CLOC) на модальное усиление для случая лазеров с одинаковой активной областью на основе 2 слоев КЯТ. Чем выше фактор оптического ограничения, тем больше параметр усиления, вычисленный на начальном (логарифмическом) участке зависимости $G$ от $J$, и тем большее модальное усиление достигается при заданном токе (рис. 5,b). В лазере CLOC с наименьшим фактором оптического ограничения параметр модального усиления $G_{0}$ на первом и втором участках зависимости $G(J)$ и максимальное усиление основного состояния составили 15,40 и $105 \mathrm{~cm}^{-1}$ соответственно. При увеличении фактора оптического ограничения па- раметр модального усиления и максимальное усиление возрастают и в лазере NWG составили соответственно 21,95 и $165 \mathrm{~cm}^{-1}$.

\section{4. Заключение}

Исследовано влияние числа слоев КЯТ в активной области и конструкции волновода на лазерные характеристики, в частности на зависимости пороговой плотности тока, дифференциальной квантовой эффективности и длины волны генерации от длины резонатора. Обнаружено, что в лазерах с таким типом активной области при увеличении потерь не происходит переключения генерации на возбужденный оптический переход, что свидетельствует об аномально низкой плотности возбужденных состояний в КЯТ, не характерной ни для КЯ, ни для КТ. Возможность достижения генерации на основном состоянии КЯТ в торцевых лазерах с предельно малой длиной резонатора и соответственно малым временем его обхода (малым К-фактором) делает такие приборы перспективными для использования в быстродействующих системах оптической связи. Также следует отметить, что использование КЯТ позволяет в широких пределах варьировать характеристики усиления. Так, при использовании 1 ряда КЯТ достигаются низкие значения плотности тока, ток прозрачности $70 \mathrm{~A} \cdot \mathrm{cm}^{-2}$ при достаточно высоком параметре усиления $14 \mathrm{~cm}^{-1}$, а для 5-10 рядов КЯТ максимальное значение модового усиления превышает $150 \mathrm{~cm}^{-1}$.

\section{Финансирование работы}

Работа выполнена при поддержке Министерства науки и высшего образования РФ, проект № 0791-2020-0002. 
А.Е. Жуков и А.М. Надточий выражают благодарность за поддержку Программе фундаментальных исследований НИУ ВШЭ в 2020 году.

\section{Конфликт интересов}

Авторы заявляют, что у них нет конфликта интересов.

\section{Список литературы}

[1] S.A. Mintairov, N.A. Kalyuzhnyy, V.M. Lantratov, M.V. Maximov, A.M. Nadtochiy, S.A. Rouvimov, A.E. Zhukov. Nanotechnology, 26, 385202 (2015). doi:10.1088/0957-4484/26/38/385202

[2] E. Moiseev, N. Kryzhanovskaya, M. Maximov, F. Zubov, A. Nadtochiy, M. Kulagina, Y. Zadiranov, N. Kalyuzhnyy, S. Mintairov, A. Zhukov. Optics Lett., 43, 4554 (2018). doi:10.1364/OL.43.004554

[3] F. Zubov, M. Maximov, N. Kryzhanovskaya, E. Moiseev, M. Muretova, A. Mozharov, N. Kaluzhnyy, S. Mintairov, M. Kulagina, N. Ledentsov, L. Chorchos, N. Ledentsov, A. Zhukov. Optics Lett., 44, 5442 (2019). doi:10.1364/OL.44.005442

[4] M.V. Maximov, L.V. Asryan, Y.M. Shernyakov, A.F. Tsatsul'nikov, I.N. Kaiander, V.V. Nikolaev, A.R. Kovsh, S.S. Mikhrin, V.M. Ustinov, A.E. Zhukov, Z.I. Alferov, N.N. Ledenstov, D. Bimberg. IEEE J. Quant. Electron., 37, 676 (2001). doi:10.1109/3.918581

[5] K.J. Beernink, J.J. Alwan, J.J. Coleman. Appl. Phys. Lett., 58, 2076 (1991). doi:10.1063/1.105015

[6] N.Y. Gordeev, M.V. Maximov, A.E. Zhukov. Laser Phys., 27, 086201 (2017). doi:10.1088/1555-6611/aa7cdc

[7] A.A. Serin, A.S. Payusov, Y.M. Shernyakov, N.A. Kalyuzhnyy, S.A. Mintairov, N.Y. Gordeev, F.I. Zubov, M.V. Maximov, A.E. Zhukov. J. Phys. Conf. Ser., 1124, 041005 (2018). doi:10.1088/1742-6596/1124/4/041005

[8] P.A. Evans, P. Blood, J.S. Roberts. Semicond. Sci. Technol., 9, 1740 (1994). doi:10.1088/0268-1242/9/9/028

[9] T.C. Newell, M.W. Wright, H. Hou, L.F. Lester. IEEE J. Select. Top. Quant. Electron., 5, 620 (1999). doi:10.1109/2944.788426

[10] M. Rossetti, P. Bardella, M. Gioannini, I. Montrosset. Proc. 14th Eur. Conf. Integrated Optics and Technical Exhibition, Contributed and Invited Papers [Eur. Conf. Integrated Photonics (Netherlands, Eindhoven, 2008)] p. 221.

\section{Impact of active region and waveguide design on performance of edge-emitting lasers based on InGaAs/GaAs quantum well-dots}

Yu.M. Shernyakov ${ }^{\mathbf{1}, 2}$, N.Yu. Gordeev ${ }^{1}$, A.S. Payusov' ${ }^{1}$, A.A. Serin ${ }^{1}$, G.O. Kornyshov ${ }^{2}$, A.M. Nadtochiy ${ }^{3}$, M. Kulagina ${ }^{1}$, S.A. Mintairov ${ }^{\mathbf{1}, 2}$, N.A. Kalyuzhnyy ${ }^{\mathbf{1 , 2}}$, M.V. Maximov ${ }^{2}$, A.E. Zhukov ${ }^{3}$

${ }^{1}$ loffe Institute, 194021 St. Petersburg, Russia

${ }^{2}$ Alferov University, 194021 St. Petersburg, Russia

${ }^{3}$ National Research University Higher School of Economics, 190008 St. Petersburg, Russia

Abstract We study edge-emitting lasers with the active area based on novel InGaAs/GaAs quantum heterostructures of transitional dimensionality referred to as quantum well-dots, which are intermediate in properties between quantum wells and quantum dots. We show that the rate of the lasing wavelength blue-shift occurring with the reduction in cavity length decreases with an increase in the number of quantum well-dot layers in the active region and the optical confinement factor. In the laser based on 10 quantum well-dot layers, the position of the lasing wavelength remains in the optical region corresponding to the emission from the ground state down to the cavity lengths as short as $100 \mu \mathrm{m}$. In the devices based on a single quantum well-dot layer and/or with low optical confinement factor, lasing directly switches from the ground state to the GaAs waveguide states omitting excited state lasing with decrease in cavity length below $200 \mu \mathrm{m}$. Such an effect has not been observed in quantum well and quantum dot lasers and is attributed to the abnormally low density of excited states in quantum well-dots.

Редактор Л.В. Шаронова 